\title{
PERFORMANCE ANALYSIS AND SIMULATION OF A GRID CONNECTED THREE-PHASE INDUCTION GENERATOR
}

\author{
Mahmoud S. Nasr \\ mahmoud111957@yahoo.com \\ Salem A. El-Saiah \\ salem_saieh@yahoo.com \\ Department of Electrical and Electronics Engineering, Faculty of Engineering, \\ University of Garyounis, Benghazi - LIBYA
}

\begin{abstract}
:
This paper aims to study the performance of a three-phase Induction Generator (IG) connected to the power utility grid. For this study, a complete analysis and simulation of a small wind power system (SWPS) based on direct modeling techniques has been presented. A theoretical analysis is reported to clarifying the system under consideration. A mathematical model associated with each component in the (SWPS) is developed. The method of interconnection has also been discussed. Finally, a finite number of disturbances associated with such wind power systems are introduced and a demonstration results such as terminal voltage, stator current, power, and speed at different modes of operation are depicted and discussed.

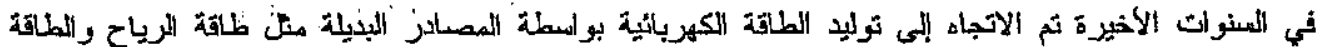

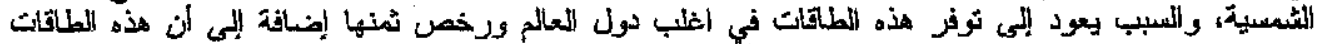

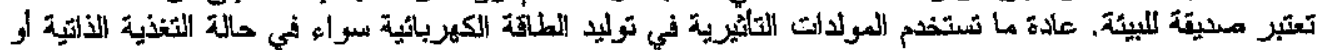
في حالة ربطها ميع الشبكة.

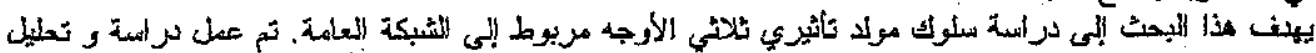

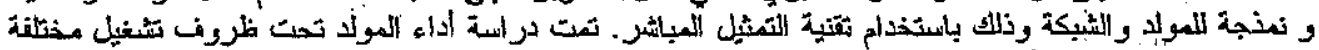

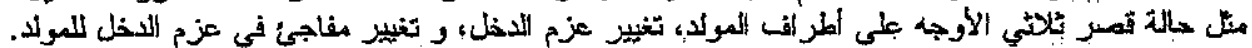

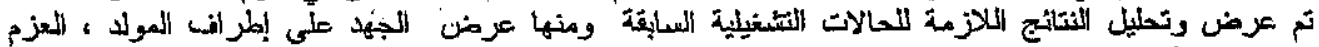

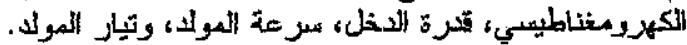

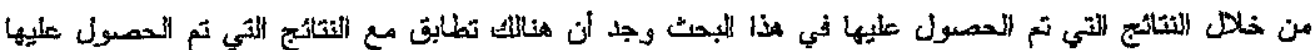

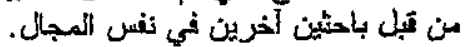

Keywords: Induction Generator (IG), Small Wind Power System (SWPS), Performance, Simulation.
\end{abstract}

\section{INTRODUCTION}

Induction Generators (IGs) have been found to be very attractive solution for alternative renewable energy tésources. In contrast to other conventional alternators, induction generators have certain inherent advantages such as, low unit cost, less maintenance, ruggedness, brushless rotor construction (squirrel cage type), high reliability during transient situations, and self protection against over loads and short circuits [1-4].

When an appropriate capacitor bank is connected across the stator terminals of an indiction machine and the rotor is driven by a prime mover, the phenomena of self-excitation will exist. Under such circumstances the terminal capacitance tends to supply the necessary lagging VARs for establishing the airgap flux and the machine is referred to as selfexcited induction generator (SEIG) [5].

Unlike the (SEIG), the frequency of the grid connected induction generator is locked to the frequency of the power utility grid but the speed may vary [6]. Capacitor banks are connected to the generator terminals to improve the system power factor and also to reduce the VARs drain from the grid to which the generator is connected. The effect of capacitors size will have to be appraised to arrive at appropriate rating.

This paper is organized as follows: Section I gives an introduction. Section II introduces the system to be investigated. Section III focused on the main components of the system under study, finally demonstrated results are presented and some specific conclusions are drawn for this paper.

\section{SYSTEM UNDER STUDY}

The system to be investigated is shown in Fig. 1. As shown, this system comprises the following components: an induction generator, power transformer, capacitor bank, static load, and short transmission line (all are three-phase). A 
mathematical model associated to each component is given below.

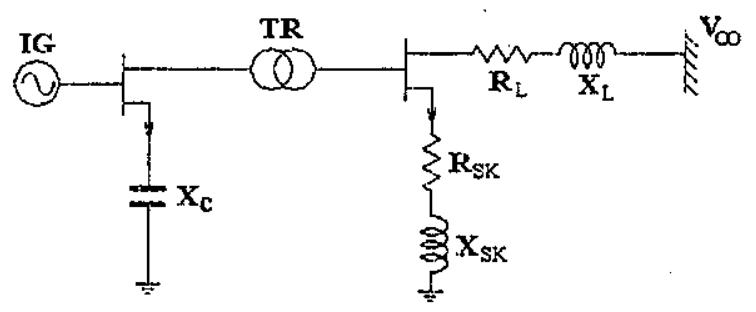

Fig. 1 Representation of a small wind power system

\section{POWER SYSTEM COMPONENTS MODELING}

In this section, only the power system components which associated with small wind power system are described.

\subsection{The induction generator}

The basic equivalent circuit of a three phase induction generator may be expressed as follows:

- Flux Linkage Equations:

In matrix notations, the flux iinkages of the stator and rotor windings, in terms of the winding inductances and currents, may be written compactly as given in (1), where subscripts $s$ and $r$ denotes to stator and rotor windings respectively [7].

$$
\left[\begin{array}{l}
\lambda_{s} \\
\lambda_{r}
\end{array}\right]=\left[\begin{array}{l}
L_{s} L_{s r} \\
L_{r s} L_{r}
\end{array}\right]\left[\begin{array}{l}
I_{s} \\
I_{r}
\end{array}\right]
$$

Where

$$
\begin{aligned}
& {\left[\lambda_{s}\right]=\left[\lambda_{a s} \lambda_{b s} \lambda_{c s}\right]^{T}} \\
& {\left[\lambda_{r}\right]=\left[\lambda_{a r} \lambda_{b r} \lambda_{c r}\right]^{T}} \\
& {\left[I_{s}\right]=\left[I_{a s} I_{b s} I_{c s}\right]^{T}} \\
& {\left[I_{r}\right]=\left[I_{a r} I_{b r} I_{c r}\right]^{T}}
\end{aligned}
$$

$$
\left.L_{s}\right\rfloor=\left[\begin{array}{ccc}
\left(L_{L s}+L_{m s}\right) & -0.5 L_{m s} & -0.5 L_{m s} \\
-0.5 L_{m s} & \left(L_{L s}+L_{m s}\right) & -0.5 L_{m s} \\
-0.5 L_{m s} & -0.5 L_{m s} & \left(L_{l s}+L_{m s}\right.
\end{array}\right]
$$

$$
\left\lfloor L_{r}\right\rfloor=\left[\begin{array}{lll}
\left(L_{t r}+L_{m r}\right) & -0.5 L_{m r} & -0.5 L_{m r} \\
-0.5 L_{m r} & \left(L_{l r}+L_{m r}\right) & \left.-0.5 L_{m r}\right) \\
-0.5 L_{m r} & -0.5 L_{m r} & \left(L_{t r}+L_{m r}\right)
\end{array}\right]
$$

$$
L_{u r}^{\text {ack }}=L_{r r}\left[\begin{array}{ccc}
\cos \theta_{r} & \cos \left(\theta_{r}+\frac{2 \pi}{3}\right) & \cos \left(\theta_{r}-\frac{2 \pi}{3}\right) \\
\cos \left(\theta_{r}-\frac{2 \pi}{3}\right) & \cos \theta_{r} & \cos \left(\theta_{r}+\frac{2 \pi}{3}\right) \\
\cos \left(\theta_{r}+\frac{2 \pi}{3}\right) & \cos \left(\theta_{r}-\frac{2 \pi}{3}\right) & \cos \theta_{r}
\end{array}\right]
$$

Lls and Lms are respectively the leakage and magnetizing inductance of the stator windings. Llr and $\mathrm{Lmr}$ are for the rotor windings. The inductance Lsr is the amplitude of the mutual inductance between stator and rotor windings.

- Voltage Equations:

Using the coupled circuit approach and generator notation, the voltage equations of the magnetically coupled stator and rotor circuits can be written as:

$\left[\begin{array}{l}\nu_{s} \\ \nu_{r}\end{array}\right]=p\left[\begin{array}{l}\lambda_{s} \\ \lambda_{r}\end{array}\right]+\left[\begin{array}{l}R_{s} \\ R_{r}\end{array}\right]\left[\begin{array}{l}I_{s} \\ I_{r}\end{array}\right]$

Where:

$\left[v_{s}\right]=\left[v_{a s} v_{b s} v_{c s}\right]$

$\left[v_{r}\right]=\left[v_{a r} v_{b r} v_{c r}\right]^{P}$

$\left[R_{s}\right]=\operatorname{diag}\left[R_{a s} R_{b s} R_{c s}\right]$

$\left[R_{r}\right]=\operatorname{diag}\left[R_{a r} R_{b r} R_{c r}\right]$

Substituting for $P[\lambda s]$ and $P[\lambda r]$ by the equation (1) and using the matrix [G] notation. Equation (9) can be written in partitioned form as:

$[\mathrm{Vs}]=[\mathrm{Rs}][\mathrm{Is}]+[\mathrm{Ls}] \mathrm{P}[\mathrm{I} \mathrm{s}]+[\mathrm{L} s \mathrm{~s}] \mathrm{P}[\mathrm{Ir}]+\omega \mathrm{H}[\mathrm{Gsr}][\mathrm{Ir}]$

$[\mathrm{Vr}]=[\mathrm{Rr}][\mathrm{Ir}]+[\mathrm{Lr}] P[\mathrm{Ir}]+[\mathrm{Lsr}] \mathrm{P}[\mathrm{Is}]+\omega \mathrm{r}[\mathrm{Grs}][\mathrm{Is}]$

Where:

$$
\begin{aligned}
& {\left[L_{r s}\right]=\left[L_{s r}\right]^{T}} \\
& {\left[G_{r s}\right]=\left[G_{s r}\right]^{P}} \\
& {\left[G_{s}\right]=\left[G_{r}\right]=0}
\end{aligned}
$$

A matrix [G] is introduced to represent the rotation performance of the electrical machine and is called the rotational inductance matrix. $\mathrm{P}[\mathrm{L}]$ is defined as $\omega r[G]$ where or is the rotor speed in electrical radians/sec. and $P$ is the differential operator.

Torque Equations:

The torque equation of the induction generator in terms of stator equations can be expressed as: 
M Nasr, S. El-Saiah, "Performance Analysis and Simulation of a Grid Connected Three-Phase Induction Generator"

$\mathrm{T}_{c}=\frac{2}{3 \sqrt{3}} \omega_{s}\left(\lambda_{s}\left(\mathrm{i}_{b s}-\mathrm{i}_{c s}\right)+\lambda_{b s}\left(\mathrm{i}_{\mathrm{cs}}-\mathrm{i}_{\mathrm{su}}\right)+\lambda_{c s}\left(\mathrm{i}_{\mathrm{ss}}-\mathrm{i}_{\mathrm{bs}}\right)\right)$

where $\omega$ is the synchronous speed in electrical radians/sec.

Also the mechanical equation of motion may be expressed as:

$\mathrm{P}(\mathrm{N}=(\mathrm{Tin}-\mathrm{Te}) / 2 \mathrm{H}$

Where Tin is the input torque and $H$ is the inertia constant for the induction machine.

\subsection{The power transformer}

The voltage equations of a three phase power transformer may expressed as:

$[V]=P[\lambda]+[R][X]$

Where

$[\lambda]=[\mathrm{L}][\mathrm{I}]$

$[\lambda]=\left[\lambda_{a 1} \lambda_{b 1} \lambda_{c 1} \lambda_{a 2} \lambda_{b 2} \lambda_{c 2}\right]^{T}$

$[v]=\left[v_{a 1} v_{b 1} v_{c 1} v_{a 2} v_{b 2} v_{c 2}\right]^{T}$

$[I]=\left[I_{a 1} I_{b 1} I_{c 1} I_{a 2} I_{b 2} I_{c 2}\right]^{T}$

$[R]=\operatorname{diag}\left[R_{a 1} R_{b 1} R_{c 1} R_{a 2} R_{b 2} R_{c 2}\right]$

$[L]=\left[\begin{array}{llllll}L_{a 1 a 1} & L_{a 1 b 1} & L_{a 1 c 1} & L_{a 1 a 2} & L_{a 1 b 2} & L_{a 1 c 2} \\ L_{b 1 a 1} & L_{b b 1} & L_{b 1 c 1} & L_{b 1 a 2} & L_{b 1 b 2} & L_{b 1 c 2} \\ L_{c \mid a 1} & L_{c 1 b 1} & L_{c t e 1} & L_{c \mid a 2} & L_{c 1 b 2} & L_{c 1 c 2} \\ L_{a 2 a 1} & L_{a 2 b 1} & L_{a 2 c 1} & L_{a 2 a 2} & L_{a 2 b 2} & L_{a 2 c 2} \\ L_{b 2 a 1} & L_{b 2 b 1} & L_{b 2 c 1} & L_{b 2 a 2} & L_{b 2 b 2} & L_{b 2 c 2} \\ L_{c 2 a 1} & L_{c 2 b 1} & L_{c 2 c 1} & L_{c 2 a 2} & L_{c 2 b 3} & L_{c 2 c 2}\end{array}\right]$

Where, subscripts 1 and 2 denote the primary and secondary quantities respectively.

\subsection{Static load.}

The lumped static load can be modeled as a resistive load Rk and a reactive load Lk. The voltage equation for the static loads may be expressed as:

$[\mathrm{Vsk}]=[\mathrm{Rsk}][\mathrm{Isk}]+[\mathrm{Lsk}] \mathrm{P}[\mathrm{Isk}]$

Where,

$\left[v_{s k}\right]=\left[v_{a k} v_{b k} v_{c k}\right]^{T}$

$\left[I_{s k}\right]=\left[I_{a k} I_{b k} I_{c k}\right]^{T}$

$\left[R_{s k}\right]=\operatorname{diag}\left[R_{a k} R_{b k} R_{c k}\right]^{r}$

$$
\left[L_{s k}\right]=\operatorname{diag}\left[L_{a k} L_{b k} L_{c k}\right]^{p}
$$

\subsection{The power factor correction capacitor}

The lumped capacitor model can be expressed by a set of differential equations as:

$[\mathrm{sc}]=[\mathrm{Csc}] P[\mathrm{Vsc}]$

Where,

$\left[I_{s c}\right]=\left[I_{a c} I_{b c} I_{c c}\right]^{T}$

$\left[v_{s c}\right]=\left[v_{a c} v_{b c} v_{c c}\right]^{T}$

$\left[C_{s c}\right]=\operatorname{diag}\left[C_{a e} C_{b e} C_{c e}\right]^{T}$

\subsection{Short transmission line}

The short transmission line can be modeled similar to the static load that described in section $\mathrm{C}$.

\section{METHOD OF INTERCONNECTION}

The method of interconnection is base on applying the Kirchhoff's current and voitage laws at each node in the system, e.g. consider the simple one node system shown in Fig. 2, which contains $m$ generators and $n$ load.

Kirchhoff's current law:

$\sum_{k=1}^{m} I_{s k g}+\sum_{e=1}^{n} I_{s m e}=0$

and Kirchhoff's voltage law may be stated as:

$\mathrm{Vsg} 1=\mathrm{Vsg} 2=\ldots .=\mathrm{V}$ sgm $=\mathrm{Vsm} 1=\mathrm{Vsm} 2=\ldots . .=\mathrm{Vsmn}$

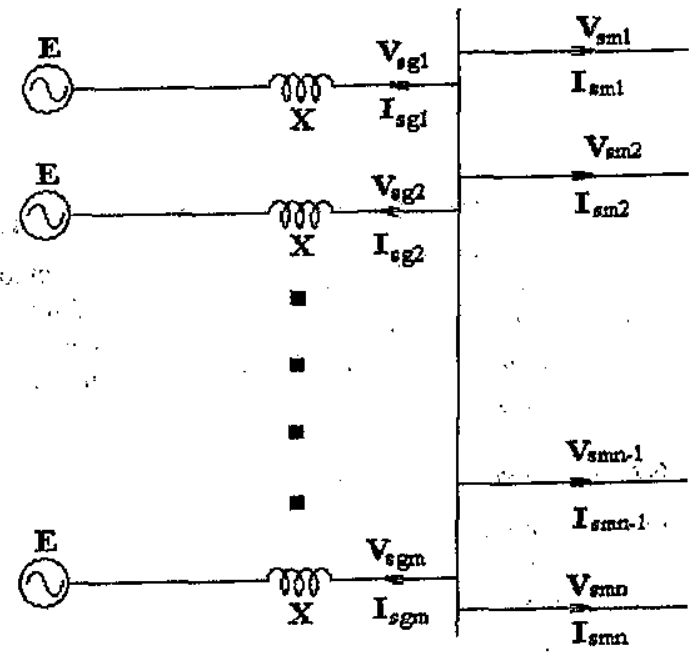

Fig. 2 Simple one node power system 


\section{REPRESENTATION OF A SMALL WIND POWER SYSTEM}

The application of the method of interconnection is illustrated by considering the simple system shown in Fig. 1, which comprises a three phase induction generator, power transformer, capacitor bank, static load, short line, and infinite busbar. 'The system can be expressed by a set of first-order differential equations of the form:

$[V]=[R][T]+[L] P[T]+[G][I]$

Where,

$[v]=\left[v_{r g}, 0,0,0, v_{\infty}, 0\right]^{r}$

$[r]=\left[I_{r g}, I_{1 T}, I_{s c}, I_{s c} I_{L} I_{s c}\right]^{T}$

$[\mathrm{R}],[\mathrm{L}]$ and $[\mathrm{G}]$ are square matrices and can be found as:

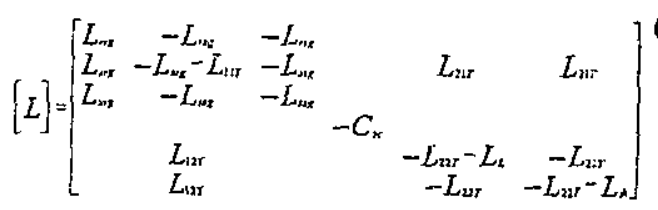

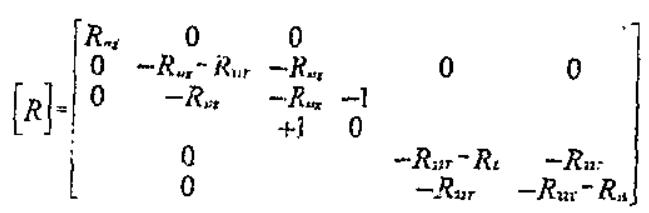

$$
\begin{aligned}
& {[G]=\left[\begin{array}{cccccc}
0 & -\omega, G_{n s} & -\omega_{r} G_{m s} & & & \\
\omega, G_{n z} & 0 & 0 & & 0 & 0 \\
\omega, G_{m z} & 0 & 0 & & & \\
& 0 & & 0 & & \\
& 0 & & & 0 & 0 \\
& 0 & & & 0 & 0
\end{array}\right]}
\end{aligned}
$$

\section{SYSTEM DISTURBANCES AND RESULTS}

A sequence of simulation studies were conducted on the system shown in Fig.1. The procedures for applying the various types of disturbances to the system under consideration ase represented in the following tasks.

\subsection{Balanced three-phase fault:}

In the simulation of balanced three-phase fault only the static impedances are replaced by fault impedances, that is:

$$
\begin{aligned}
& \text { Rask }=\text { Rbsk }=R \operatorname{csk}=R_{f} \\
& \text { Lask }=\text { Lbsk }=\text { Lcsk }=L_{f}
\end{aligned}
$$

Where $R_{f}$ and $L_{f}$ are the resistance and inductance of the fault impedance.

Generator synchronized to the system with ( 0.9 p.u.) constant torque and three phase balanced fault applied at the transformer HV winding after two seconds. After $200 \mathrm{~ms}$ the fault is cleared.

Figures 3, 4, 5, 6, and 7 show waveforms rotor speed, electromagnetic power, power input, average terminal voltage, and phase A current respectively.

\subsection{Cycling in input torque:}

A $25 \%$ of the rated input torque $(0.9$ p.u.) as a cycling torque superimposed on the $(0.75)$ of the rated constant input torque. The equation of the input torque for $1 \mathrm{~Hz}$ frequency of cycling can be expressed as:

Tin $=\left(0.75^{*} 0.9\right)+\left(0.25^{*} 0.9\right) \sin (2 \pi \mathrm{T})$

Selected results of the study are given in figures 8,9 , 10,11 , and 12 .

\subsection{Step change in input torque:}

The input torque is made equal to (0.25p.u.) for the first two seconds of the simulation and the input torque is increased to (0.9p.u.) and kept constant for the rest of the simulation time.

Selected results represent rotor speed, electromagnetic power, power input, average terminal voltage, and phase A current are, respectively given in figures $13,14,15,16$, and 17 .

\section{CONCLUSIONS}

In this paper a study, modeling, and analysis of a small wind power system has been presented. The system considered in this paper consists of an induction generator, power transformer, short transmission line, static load, and a power factor correction capacitor. In order to examine the mentioned system, a multi modes of operation and disturbances; which are practically sudden; are applied such as three phase short circuit, cycling in input toraue, and step change in input toque, these modes are simulated and good correlarions to the practical situations are depicted. The simulation and fault duration used in the research respectively; are 5 $\mathrm{sec}$, and $200 \mathrm{msec}$, which are really recommended time for the most authors in the field.

\section{ACKNOWLEDGEMENT}

The authors would like to express their deeply appreciations to professor Dr. Mohammed $\mathrm{S}$. Bumuad, professor of electrical drives at the Electrical Engineering department, Garyounis University for his valuable assistance during the realization of the presented research. 


\section{REFERENCES}

[1] S. S. Murthy B.P.Singh, B.Singh and A. Gorashi "Analysis of Wind Driven Grid Connected Induction Generators Under Unbalanced Grid Conditions" IEEE trans. On Energy Conversion, Vol. 9, no. 2, pp. 217-223, June 1994.

[2] E. Bim et al. "Voltage Compensation of an Induction Generator with Long Shunt Connection" IEEE Trans. On Energy Conversion, Vol. 4, no. 3, pp. 526-530, Sept. 1989.

[3] M. S. Buamud, M.S. Nast and S. A. El-Saiah, "A Proposed Discrete Model for Three-Phase Induction Generator" $5^{\text {th }}$ International Engineering Conference, Mansoura University, Egypt, pp. 45-56, 2006.

[4] S.S. Murthy, B.P. Singh, C. Nagmani, and K.Satyanarayana "Studies on the Use of Conventional Induction Motors as Self-Excited Induction Generators" IEEE Trans. On Energy Conversion, Vol. 3, no. 4, pp. 842-848, 1988.

[5] J.Elder et al. "Self-Excited Induction Machine As Low Cost Generator" IEE proceedings, Pt. C, Vol.131, no. 2, pp 33-40, 1984.

[7] Chee. Mung Ong "Dynamic Simulation of Electric Machines Using Matlab/Simulink" Book, McGraw Hill Publications, 1998.

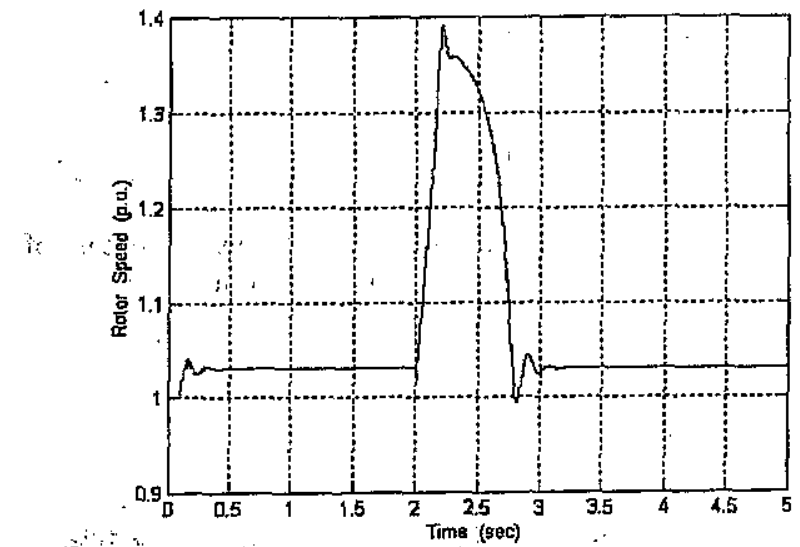

Tig 3 Rotorsped vis time, case of three-phase short cingut

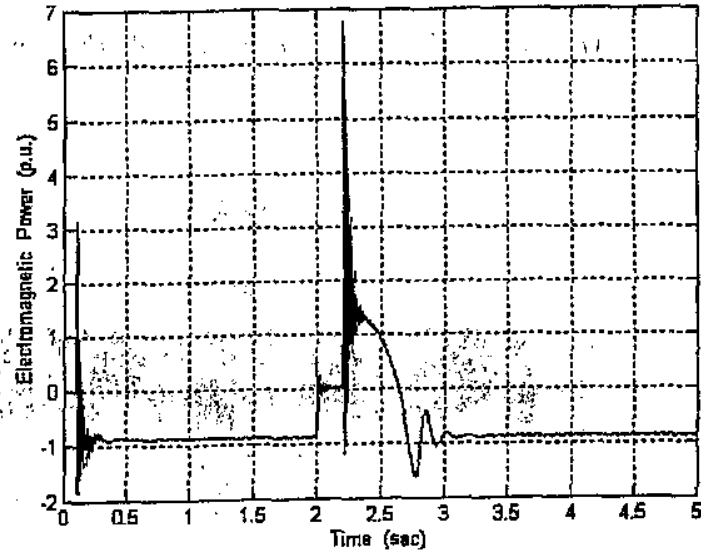

Fig.4 Electromagnetic power vs time, case of threephase short circuit

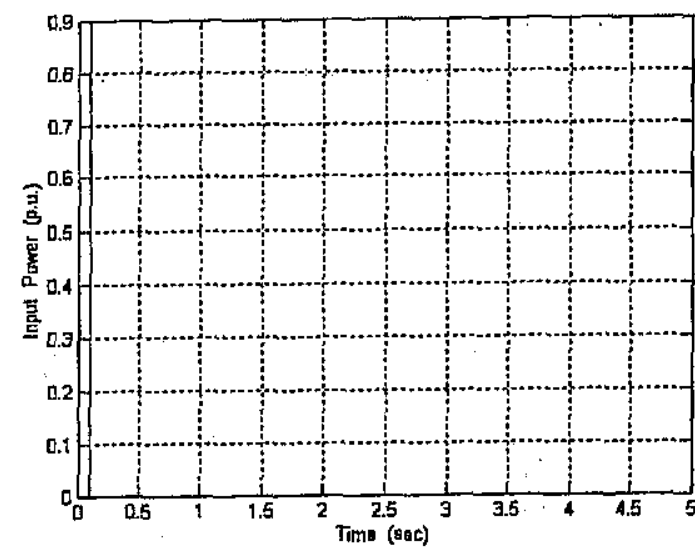

Fig. 5 Power input vs time, case of three-phase short circuit

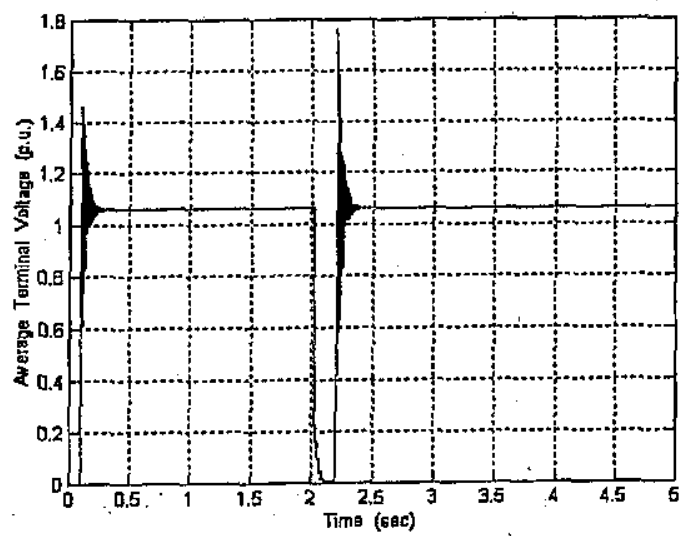

Fig. 6 Average terminal voltage vs time, case of three-phase short circuit 


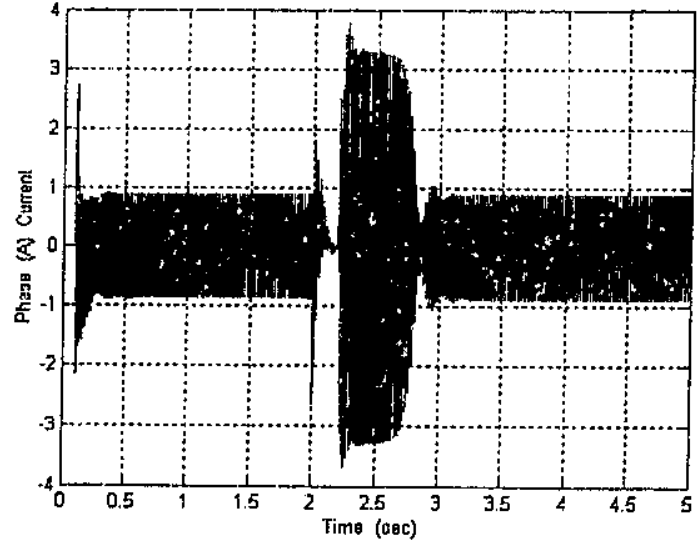

Fig. 7 Phase (A) current vs time, case of three-phase short circuit

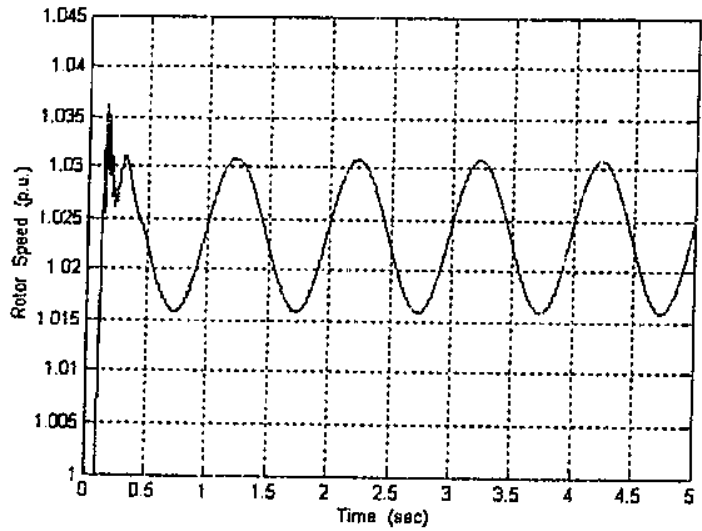

Fig. 8 Rotor speed vs time, case of cycling in input torque

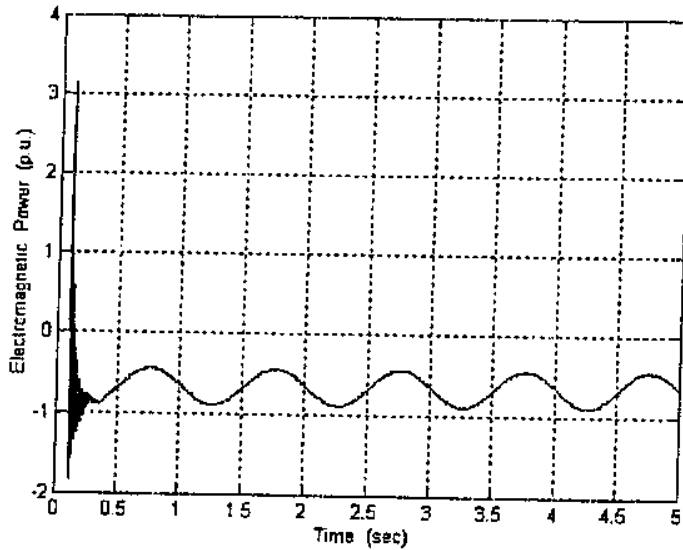

Fig. 9 Electromagnetic power vs time, case of cycling in input torque

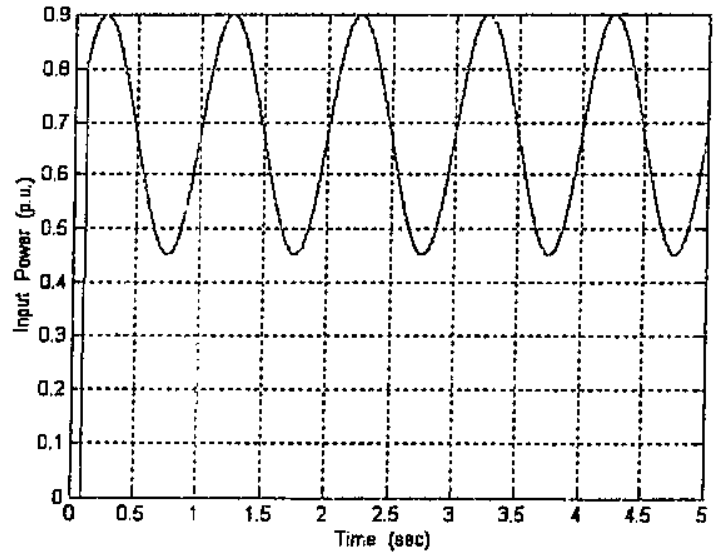

Fig. 10 Power input vs time, case of cycling in input torquse

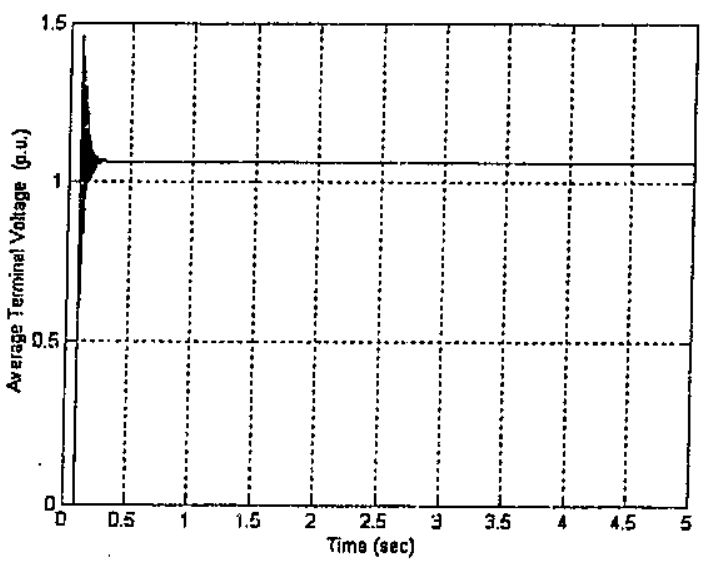

Fig. 11 Average terminal voltage vs time, case of cycling in input torque

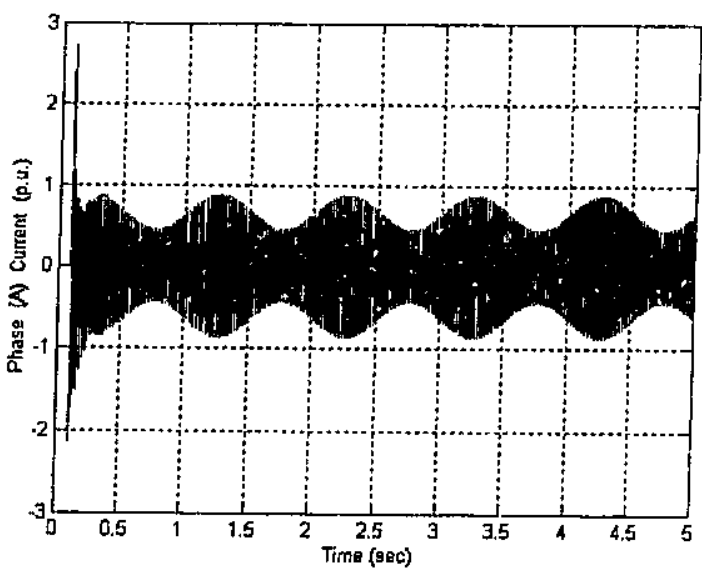

Fig. 12 Phase (A) current vs time, case of cycling in input torque 


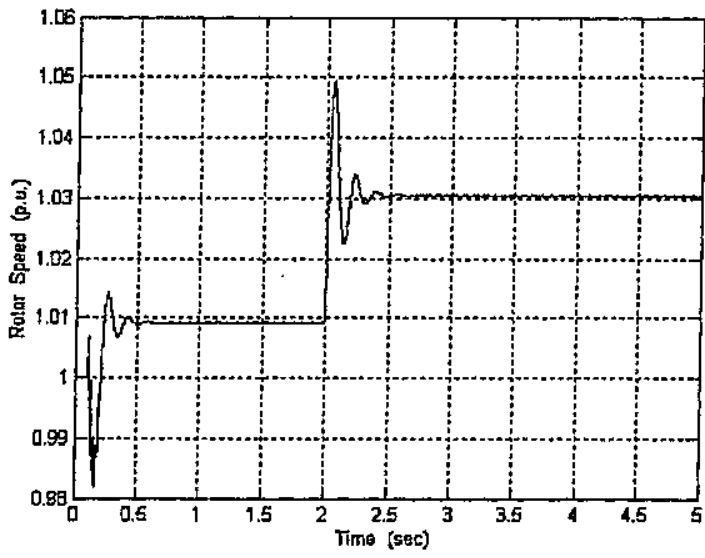

Fig. 13 Rotor speed vs time, case of step change in input torque

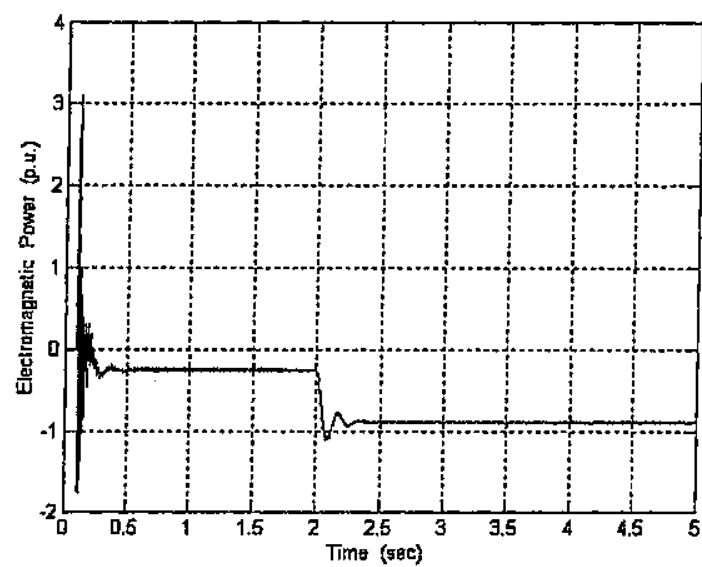

Fig. 14 Electromagnetic power vs time, case of step change in input torque

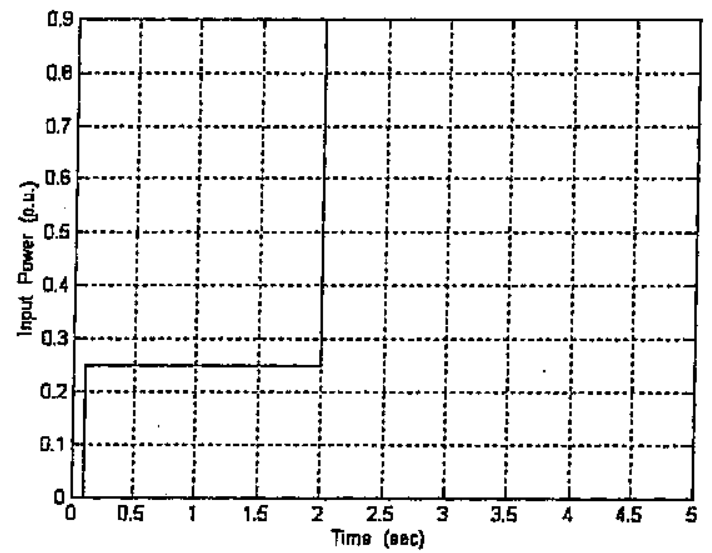

Fig. 15 Power input vs time, case of step change in input torque

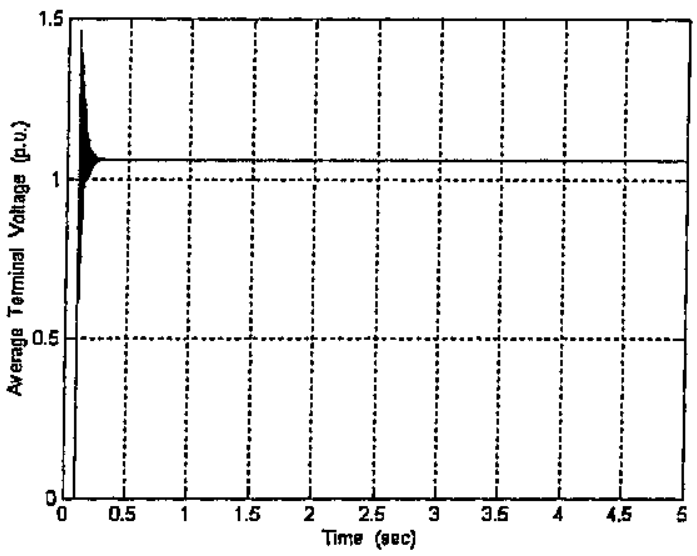

Fig. 16 Average terminal voltage vs time, case of step change in input torque

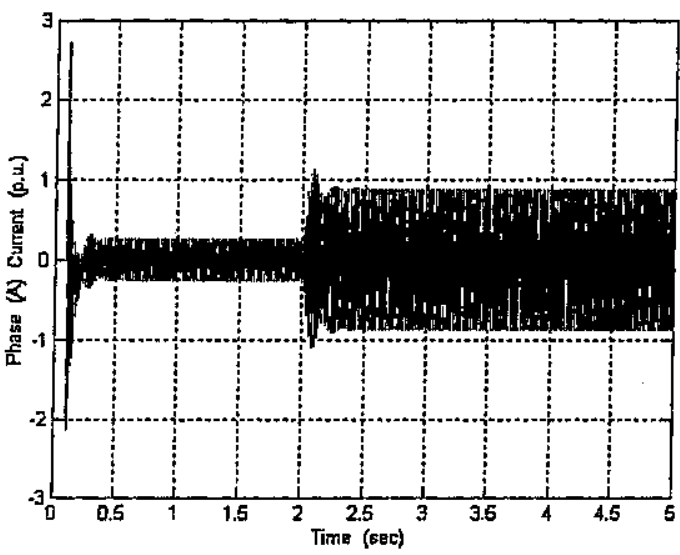

Fig. 17 Phase (A) current vs time, case of step change in input torque 


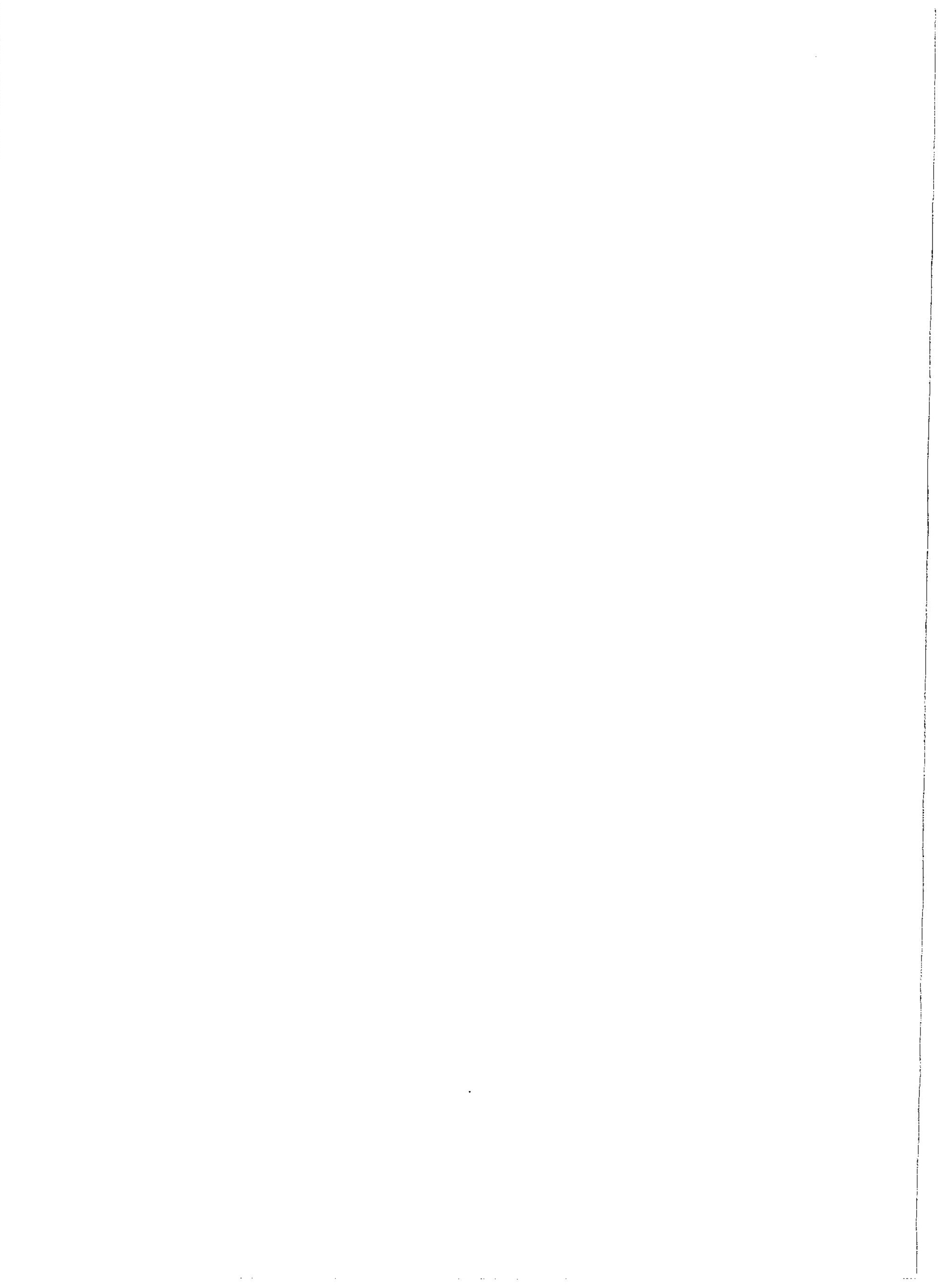



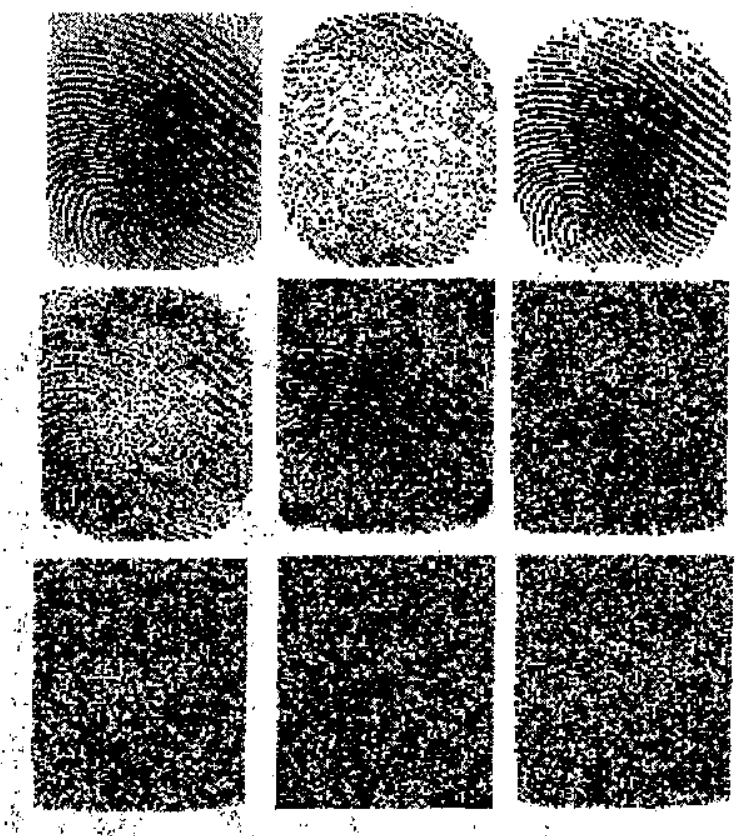

Figure 3 Multi-grey level image and its 8 binary

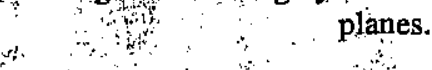

s.

This method called the bit plane decomposition technique decodes a given pixel value within the originalitmage using the following equation:

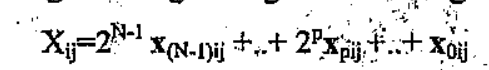

where $x_{(N-1) i j}, x_{p i j}, x_{0 i j}$ are either 0 or 1 and represent the pixel values corresponding respectively to the $\mathrm{N}$ binary planes at position $(i, j)$ for all $i, j \in\{0, \ldots, 511\}$ (case of 512 by 512 image resolution) and $N=\log _{2} M$, $M$ being the number of grey-levels.

\subsection{Hịgh Resolution Images}

In general, increasing the image resolution requires larger demand on the memory capacity. To overcome this limitation, we split the image into a set of non-overlapping sub-images. Then we dedicate one pyramidal net to handle each sub-image. This allowed us, in the case of a classification problem, to define what we called the convergence map that was used to discard the irrelevant sub pyramids (i.e. do not converge) and build the whole pyramid using only the output of sub-pyramids that converged, reducing the memory storage required for a high resolution image with the added advantage of being able to train all sub-pyramids in parallel if necessary. The tests were performed on a four pattern classification problem convergence map highlighting the parts of the images which are totally similar (grey parts), totally different (low values) or in between (values greater than 0.1 ).

\section{THE PROPOSED SAMPLING SCHEME}

The manner with which the n-pixels are extracted to form the input to the $p \mathrm{RAM}$ node is referred to the input sampling or mapping. These combined pixels form the sub-pattern, called n-tuple state, generally used as an address to a single $p \mathrm{RAM}$, to decode the corresponding $n$-tuple state. we propose a new input mapping based on data analysis as we believe that extracting knowledge from the actual data to select the $n$-tuples is the most appropriate way to extract the salient features of the image.

In order to obtain the input mapping in the case of a $P$ pattern recognition problem, we consider the local variations seen by each pixel within the image according, to the data used: As a result, we get $\mathrm{L}$. (innage size in pixels) functions each of which can be represented by $P$ (number of patterns) strings of length $\mathrm{T}$ (size of each training set). Since each individual pixel goes through a cycle of variations, we suggest to compute the resultant probability of a given $n$-tuple to be in a given state based on the individual probability densities of the constituent pixels. Hence, we use the frequency of occurrence of a pixel to be in the state 1 , as the main metric to represent changes at the pixel level while handling one training set at a time. Normally, if a n-tuple state is a characteristic one for a given training set, this state should be the pixel level while handling one training set at a time. Normally, if a n-tuple state is a characteristic one for a given training set, this state should be the frequency of occurrence of a pixel to be in the state 1 , as the main netric to represent changes at the pixel level while handling one training set at a time. Normally, if an n-tuple state is a characteristic one for a given training set, this state should be approximately constant (i.e. occurs with a high probability) and that particular state must have a low probability to occur for other training sets. Hence, to group pixels in n-tuples we must test for their frequency of occurrence in a particular state.

\section{FINGERPRINT RECOGNITION}

Fingerprint recognition is one of the most successful visual biometric applications which can be carried out by having a fingerprint scanned. The data from the scan is converted to a unique template, encrypted, and stored as numerical data. The recognition requires the individual to present his fingerprint for comparison with the previously recorded data. A fingerprint recognition scheme looks at the patterns found on a fingertip, for instance, we can use certain distinguishing features such as the ridges and valleys found on the surface tips of a human finger to identify an individual. The problem that one can face is the feature extraction stage on which we base the recognition. We propose, as an aiternative to classical 
techniques, the use of a pRAM neural net with a judiciously seiected data zased input mapping to overcome the probiem of feature extraction stage.

The fingerprint is $192 \times 128$ multi-grey level image, so we convert it to binary images using the previously stated method and we divide the resultant images into sinall shrinks each of which has a size of $24 \times 16$, as shown in Figure 3.

\section{TESTS AND RESULTS}

A number of computer simulations were carried out to highlight the effect of using different input mappings on a pyramidal neural network to perform recognition of $24 \times 16$ binary inages. Three input rnappings were tested, namely the structured (i.e. regularly sampies n consecutive pixels: linear), the database was derived using the proposed algorithm given in the previous section, and a permuted version of the latter. The spatial distributions of the n-tuples represented by these mappings are respectively indicated using the joint n-tuple distance map given in Fig 5 .

This figure also summarizes the proportion of each n tuple type obtained from a corresponding n-tuple input mapping. The performance is measured using the convergence and recognition rates, as these are the most common metrics used for pattern recognition problems. The obtained results are illustrated in Fig.4.

Figure 4 shows that the network's convergence is better in the case of the permuted and data-based mappings when compared to linear mapping. Using the permuted mapping we managed to achieve low convergence error rate approximated at $2 \%$ compared to $13 \%$ for the structured mapping and $6 \%$ in the case of the data-based one without permutation.

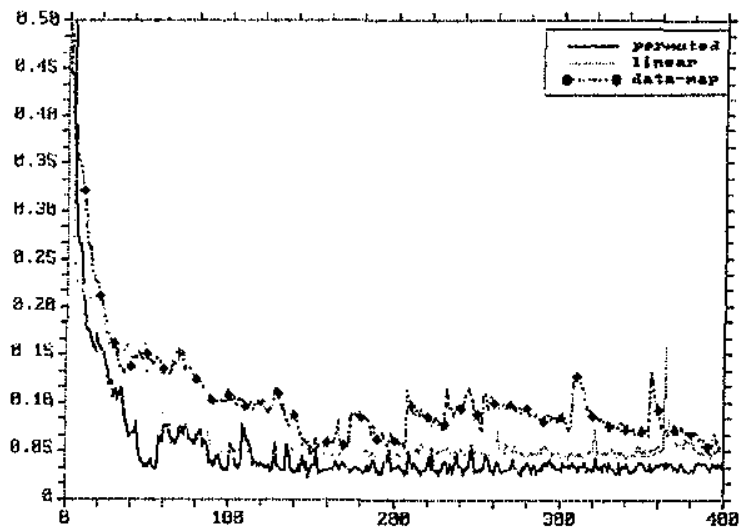

Fig.4 Recognition error function of the number of pRAM net iterations for three different mappings

The fluctuations seen are the result of changes in the $p$ RAM memory contents which vary in the range
[0,1]. The network using the permuted mapping achieved the highest recognition rate of $93 \%$, whereas the non-permuted mapping only achieved $80 \%$ even worse than that of the structured input mapping. This result can be justified by consulting the JND map.

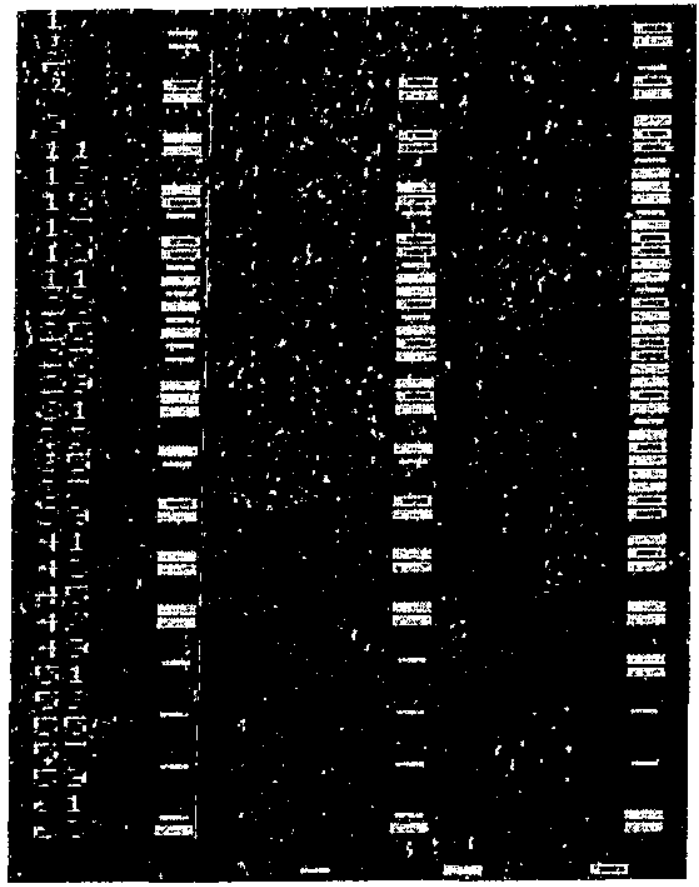

Fig. 5. The Joint n-tuple Distance (JND) map

Indeed, we see that the structured mapping presents relatively good distribution of all n-tuple types throughout the input layer of the pyramidal net compared to the data-based input mapping In rearranging the n-tuples obtained, using the databased algorithm, we obtain the permuted mapping, which presents better spatial distribution of the $n$ tuples compared to the original data-based one. This result allows us to state that breeding a good input mapping is not sufficient when used with a $p R A M$ pyramidal network, we must, in addition, provide a uniform distribution of the n-tuple types at the network input.

The bit plane encoding scheme does not assume any underlying constraints on the images and allows the use of the same $p$ RAM net to exercise different types of data i.e. the binary planes corresponding to each grey scale image, which makes the computer simulation much more easier. As a result, the obtained pRAM net was extensively exercised to handle successive fingerprint images using the transformations stated above which required a total of 512 pRAM nets in order to perform recognition of fingerprints. However, we were faced with some 


\section{S. Al-Ghamdi, "Fingerprint Recognition Based Upon A Data Analysis Input Mapping for a pRAM Neural Network"}

redundancy in the obtained binary parts, the reason why we used the convergence map to reduce the number of pRAM nets considered in our simulation. The work is still going on, and the obtained results will be published in the near future.

\section{CONCLUSION AND FURTHER WORK}

In this paper $p$ RAM nets were simulated and used to tackle an image recognition problem. This study emphasized the benefit in using a $p$ RAM net with a two stage input mapping based on data analysis. The first stage extracts n-tuples according to discrimination among patterns at the n-tuple level. This delivers a mixture of n-tuple types and when used with a single pyramidal $p$ RAM net without any overlap among n-tuples, it required an adequate distribution of the obtained n-tuples at the pyramid base. The application of these propositions to binary image recognition was successful and confirmed their effectiveness when handling a pattern recognition task. The obtained results are very motivating and encouraging. This work is still under investigation in order to set up a complete automatic system to handle recognition of fingerprints. We are also investigating the implementation of a dynamic reconfigurable $p$ RAM net with a complete test bench software based on Java programming so that we cant apply multithreading to use several nets simulator and run them in parallel. This will certainly improve the processing speed of the recognition task handled. The use of more than one pyramid, so as to allow for the overlap among the n-tuples, plays a positive role in enhancing the performance. Compression can be performed using state of the art techniques to the input image instead of applying it as it is, in a raw format, to the input of the pRAM.

These suggestions are left as a possible continuation to this work. However, we should bear in mind that this application is meant to be a tool to verify the adequacy of the proposed approach to select adequate n-tuples and the effect of distributing them at the input of a Boolean neural network. We did not intend to specifically solve the fingerprint recognition problem, this is the reason why no complete comparative study was undertaken with other techniques in this paper. Nevertheless, the good results obtained are very motivating and suggest that the use of such a scheme should be further investigated.

\section{REFERENCE}

[1] T. G. Clarkson, The $p$ RAM as a hardware realisable neuron, Proceedings Neural Networks, 1992 pp. 140-146.

[2] M. Ouslim, Analysis of the n-tuple technique based upon two perspectives to determine n-tuple groupings, $\mathrm{PhD}$ thesis, University of Nottingham, 1997.

[3] $M$. Ouslim and $K . M$. Curtis, $\mathbf{P}$ pattern recognition based on a probabilistic RAM net using n-tuple input mapping, IEE Proc. Vis. Image, Signal Process., Vol. 145 No. 6, Dec. 1998, pp. 415-420.

[4] M. Ouslim and Saleh. A. Alghamdi, Enhancing the main properties of the $p$ RAM neural network. $7^{\text {th }}$ annual IEEE technical exchange meeting, April 2000, Saudi Arabia. 